TRANSACTIONS OF THE

AMERICAN MATHEMATICAL SOCIETY

Volume 351, Number 6, Pages 2197-2212

S 0002-9947(99)01949-2

Article electronically published on March 1, 1999

\title{
TOWARDS A HALPHEN THEORY OF LINEAR SERIES ON CURVES
}

\author{
L. CHIANTINI AND C. CILIBERTO
}

\begin{abstract}
A linear series $g_{\delta}^{N}$ on a curve $C \subset \mathbf{P}^{3}$ is primary when it does not contain the series cut by planes. For such series, we provide a lower bound for the degree $\delta$, in terms of $\operatorname{deg}(C), \mathrm{g}(C)$ and of the number $s=\min \{i$ : $\left.h^{0} \mathcal{I}_{C}(i) \neq 0\right\}$. Examples show that the bound is sharp. Extensions to the case of general linear series and to the case of curves in higher projective spaces are considered.
\end{abstract}

\section{INTRODUCTION}

Let $C$ be a smooth, complete curve of genus $g$ over an algebraically closed field $k$ of characteristic zero. If $C$ has a line bundle $\mathcal{L}$ generated by global sections, of degree $d$ with $h^{0} \mathcal{L}=r+1$, i.e. a complete, base point free $g_{d}^{r}$, determining a morphism of $C$ to $\mathbf{P}^{r}$ which is birational onto its image $\Gamma$, then the classical theory of Castelnuovo provides a bound for the genus $g$ of $C$ in terms of $d$ and $r$. Indeed, one has

$$
g \leq p_{a}(\Gamma) \leq G(r ; d):=\left(\begin{array}{c}
m \\
2
\end{array}\right)(r-1)+m \epsilon,
$$

where $d=m(r-1)+\epsilon$ with $0 \leq \epsilon \leq r-2$ and this bound is sharp (see [H2]).

A natural question in this circle of ideas is to look for a similar sharp upper bound $G\left(r_{1}, \ldots, r_{n} ; d_{1}, \ldots, d_{n}\right)$ for the genus of a curve $C$ having several different linear series $g_{d_{i}}^{r_{i}}, i=1, \ldots, n$. This question has been considered by Accola in [A] and it can be solved, in some cases, with the same techniques used to prove Castelnuovo's bound (!).

Another classical direction in which Castelnuovo's theory has been developed has been the search for a sharp upper bound $G(r ; d, s)$ for the genus of a nondegenerate curve of degree $d$ in $\mathbf{P}^{r}$ not lying on any irreducible surface $S$ of degree $\sigma<$ s. This is called the Halphen problem, inasmuch as it was first considered by Halphen in a famous memoir of 1882 (see [Ha], and, for more recent references, $[\mathrm{H} 2]$ and [CCD1]). Therefore, taking into account Accola's viewpoint mentioned above, a natural development of both Castelnuovo's and Halphen's theories consists in looking for similar bounds for the genus of nondegenerate curves of degree $d$ in $\mathbf{P}^{r}$ not lying on any irreducible surface $S$ of degree $\sigma<s$ and having the further property of possessing an additional linear series $g_{\delta}^{N}$, besides the $g_{d}^{r}$ cut out by the hyperplanes of $\mathbf{P}^{r}$. This is the question we deal with in the present paper, which is essentially devoted to setting the foundations of this Castelnuovo-Halphen theory

Received by the editors February 22, 1996.

1991 Mathematics Subject Classification. Primary 14H50.

(C)1999 American Mathematical Society 
for curves with extra series and to studying a few relevant examples. Hence this note can be seen as a continuation of the mentioned papers [A], [CCD1] and [H2] as well as of other papers like $[\mathrm{B}],[\mathrm{C} 1],[\mathrm{CK}],[\mathrm{CL}],[\mathrm{L}],[\mathrm{M}],[\mathrm{P}]$, etc., in which questions like the ones we consider here are dealt with in some particular instances.

We recall that Castelnuovo's (Halphen's) classical theory is based on the idea of bounding from below the Hilbert function $h_{\Gamma}(n)$ of an irreducible, nondegenerate curve $\Gamma$ in $\mathbf{P}^{r}$ (not lying on any surface of degree $\sigma<s$ ), or, if one wishes, in bounding from below the function $h^{0} \mathcal{O}_{C}(n H)$, where $C$ is, as above, the normalization of $\Gamma$ and $H$ is the pull-back to $C$ of a hyperplane in $\mathbf{P}^{r}$. In the same vein, our approach here consists in trying to bound from below the twisted Hilbert function $h^{0} \mathcal{O}_{C}(D+n H)$, where $|D|$ is the extra linear series on $C$. Our main results in this direction are contained in $\S 2$, while $\S 1$ is devoted to generalities and to preparatory material. In particular we note our descent lemmas 2.3 and 2.4, which play an essential role in our approach and whose proofs are consequences of previous results we proved (in collaboration with Di Gennaro) in [CCD1] and [CCD2]. As in the classical theory, this sort of descent lemma would be almost useless if not accompanied by a numerical analysis aimed to find the optimal function minimizing the twisted Hilbert functions of the curves we deal with. This numerical analysis is carried out in $\S 3$ and, as sometimes happens also in the classical case (see [CCD2]), it is the hardest part of the whole story. The subsequent sections are devoted to applications. First we concentrate in $\S 4$ on space curves, i.e. curves in $\mathbf{P}^{3}$, to which theorem 2.3 applies. Our main results (see proposition 4.1 and theorem 4.4 ) give a lower bound for the degree $\delta$ of a $g_{\delta}^{N}$ on a curve of degree $d$ and genus $g$ not lying on any surface of degree $\sigma<s$ in $\mathbf{P}^{3}$, under the additional hypothesis that the series is primary, i.e. it does not contain the series cut out by the planes. The bound is in terms of $d, g$ and $s$, and it is sharp. In particular we are able to bound the gonality and the degree of a linear series of dimension 2, on curves of certain types like complete intersections, Halphen curves, curves on quadrics, etc., and to classify those curves for which the bound is attained. As we indicated before, some results of this type can already be found in the papers we mentioned above. We are also able to give information for the non-primary series, but in this case the numerical analysis needed for producing sharp bounds becomes more complicated, and will not be developed here. Finally in $\S 5$, using proposition 2.4, we make applications to curves in higher projective spaces. Our results in this case are not sharp as for space curves; however, we are able to provide another proof of Accola's bound for the gonality of Castelnuovo's curves, and we give also a lower bound for the degree of a $g_{\delta}^{N}$ with $N \geq 2$ on such curves; this bound is sharp in some numerical range for $d, N$.

We believe that the method presented here for the study of extra linear series can be usefully applied in many other circumstances: it will be enough to use the results of $\S 2$ with an appropriate numerical analysis. This paper is essentially devoted to introducing the method, showing its first application and proving a few results for some specific classes of curves, most of which are new, and, we feel, not without some interest.

\section{Notation AND PRELIMINARIES}

Let $\Gamma \subset \mathbf{P}^{r}$ be a projective irreducible nondegenerate curve of degree $d$ and let $\sigma: C \rightarrow \Gamma$ be its normalization. Let $g$ be the genus of $C$. Let $\omega_{C}$ be the canonical sheaf on $C$ and let $H$ be the pull-back on $C$ of the hyperplane divisor of $\Gamma$. 
For any divisor $D$ on $C$, we denote by $\mathcal{O}_{C}(D)$ the sheaf of sections of the line bundle associated to $D$, and $\delta$ will denote the degree of $D$.

Definition 1.1. The Hilbert function of $D$ with respect to $H$ is the function

$$
\phi_{D}(n)=h^{0} \mathcal{O}_{C}(D+n H) .
$$

We say that $D$ is $H$-primary if it is effective and $h^{0} \mathcal{O}_{C}(D-H)=0$, i.e. if $\phi_{D}(n)=0$ for $n<0$ while $\phi_{D}(n)>0$ when $n \geq 0$.

$\Delta_{D}^{1}$ and $\Delta_{D}^{2}$ will denote the first and the second difference of $\phi_{D}$, e.g.

$$
\begin{gathered}
\Delta_{D}^{1}(n)=\phi_{D}(n)-\phi_{D}(n-1), \\
\Delta_{D}^{2}(n)=\Delta_{D}^{1}(n)-\Delta_{D}^{1}(n-1)=\phi_{D}(n)-2 \phi_{D}(n-1)+\phi_{D}(n-2) .
\end{gathered}
$$

Clearly

$$
\phi_{D}(n)=\sum_{i=-\infty}^{n} \sum_{j=-\infty}^{i} \Delta_{D}^{2}(j)
$$

and for $n \ll 0$ we have $\phi_{D}(n)=\Delta_{D}^{1}(n)=\Delta_{D}^{2}(n)=0$.

It follows by the definition that $\phi_{D}(n+1)>\phi_{D}(n)$ whenever $\phi_{D}(n+1)>0$.

When $\Gamma$ is smooth and arithmetically normal, then, if we take $D=0, \phi_{D}$ turns out to be the usual Hilbert function of $\Gamma=C$.

Definition 1.2. Define the level (of speciality) of $D$ with respect to $H$ as the number

$$
e(D)=\max \left\{n: h^{1} \mathcal{O}_{C}(D+n H)>0\right\} .
$$

The number $e:=e(0)$ is also called the level of $C$. The divisor $D$ is non-special if and only if $e(D)<0$. If $D$ is effective, then $e(D) \leq e$.

Remark 1.3. If $D$ is $H$-primary, then

$$
\phi_{D}(0)=\Delta_{D}^{1}(0)=\Delta_{D}^{2}(0)=h^{0} \mathcal{O}_{C}(D) .
$$

For $n>e(D)$, by Riemann-Roch we have $\phi_{D}(n)=n d+\delta-g+1$.

For $n>e(D)+1$ we have $\Delta_{D}^{1}(n)=d$.

For $n>e(D)+2$ we have $\Delta_{D}^{2}(n)=0$.

Conversely, we have:

Proposition 1.4. Assume $\phi_{D}(n)=n d+\delta-g+1$. Then $n>e(D)$.

Assume $\Delta_{D}^{1}(m)=d$ for all $m \geq n$. Then $n>e(D)+1$.

Assume $\Delta_{D}^{2}(m)=0$ for all $m \geq n$. Then $n>e(D)+2$.

Proof. The first assertion follows by Riemann-Roch. The second and the third are easy consequences of the first.

Proposition 1.5. Put $\mathcal{O}_{C}\left(D^{\prime}\right) \simeq \omega_{C} \otimes \mathcal{O}_{C}(-D)$. Then

$$
\Delta_{D}^{2}(i)=\Delta_{D^{\prime}}^{2}(2-i)
$$

for all $i$.

Proof. Follows by Serre duality.

Proposition 1.6. $\Delta_{D}^{1}$ is nondecreasing, i.e. $\Delta_{D}^{2}(n) \geq 0$ for all $n$. 
Proof. Let $Z$ be a general hyperplane section of $C$ and look at the exact commutative diagram

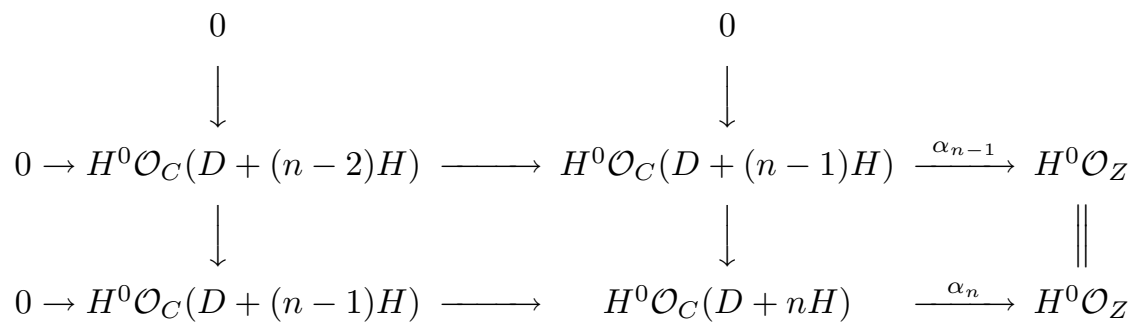

in which $\Delta_{D}^{1}(n-1)=\operatorname{rank}\left(\alpha_{n-1}\right)$ and $\Delta_{D}^{1}(n)=\operatorname{rank}\left(\alpha_{n}\right)$.

\section{BASIC RESUlts}

We collect here some basic lemmas which will be necessary for our numerical analysis of the Hilbert function of $D$.

While the speciality lemma is well-known, the descent lemmas are new and useful.

Proposition 2.1 (Speciality Lemma). Let $\Gamma$ be a curve in $\mathbf{P}^{r}, \sigma: C \rightarrow \Gamma$ its normalization, of genus $g$, let $|D|$ be a base point free linear series on $C$, and put $N+1=h^{0} \mathcal{O}_{C}(D)$. Then $e(D) \leq e \leq \delta-N-1$.

If $e=\delta-N-1$, then either $e \leq 0$ and $g \leq 1$, or the image on $\Gamma$ of the general divisor of $|D|$ lies on a line and either $N=1$ or $N=r=2$.

If $e=\delta-N-2$, then either $e \leq 0$, or the image on $\Gamma$ of the general divisor of $|D|$ lies on a plane.

Furthermore, if $D_{0} \in|D|$ is a general divisor and $\mathcal{I}$ is its ideal sheaf in the projective space $\mathbf{P}^{r}$, then $h^{1} \mathcal{I}(e) \geq N$.

Proof. Since $|D|$ is base point free, we may assume $D_{0} \in|D|$ disjoint from the pull-back of the singular locus of $\Gamma$.

The exact sequence $0 \rightarrow \mathcal{I}_{D_{0}, C}\left(\omega_{C}\right) \rightarrow \mathcal{O}_{C}\left(\omega_{C}\right) \rightarrow \mathcal{O}_{D_{0}} \rightarrow 0$ gives in cohomology

$$
H^{0} \mathcal{O}_{C}\left(\omega_{C}\right) \rightarrow H^{0} \mathcal{O}_{D_{0}} \rightarrow H^{1} \mathcal{I}_{D_{0}, C}\left(\omega_{C}\right) \rightarrow H^{1} \mathcal{O}_{C}\left(\omega_{C}\right) \rightarrow 0
$$

where $h^{1} \mathcal{I}_{D_{0}, C}\left(\omega_{C}\right)=h^{1} \mathcal{O}_{C}\left(\omega_{C}(-D)\right)=h^{0} \mathcal{O}_{C}(D)=N+1$, so that the image of the first map has dimension $\delta-N$.

On the other hand, since $h^{0} \omega_{C}(-e H)>0$, we have a factorization

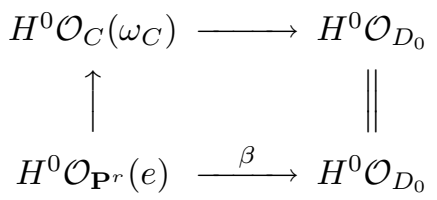

so that $\operatorname{dim}(\operatorname{Im}(\beta)) \leq \delta-N$, which means $h^{1} \mathcal{I}(e) \geq N$.

Finally, for any set of distinct points in $\mathbf{P}^{r}$, we have $\operatorname{dim}(\operatorname{Im}(\beta)) \geq e+1$, and, if $e \geq 1$, equality holds if and only if $D_{0}$ lies on a line, while $\operatorname{dim}(\operatorname{Im}(\beta))=e+2$ implies that $D_{0}$ is contained on a plane.

More generally, $\operatorname{Im}(\beta)$ has dimension $\gg e$, i.e. $\delta-N-1 \gg e$, unless the points of $D_{0}$ belong to some linear subspace.

In particular: 
Corollary 2.2. If $C$ is hyperelliptic then $e=0$.

If $C$ is trigonal, then $e \leq 1$, and $e=1$ if and only if the image on $\Gamma$ of the general divisor of the $g_{3}^{1}$ spans a line.

If $C$ is quadrigonal, then $e \leq 2$, and $e=2$ implies that the image on $\Gamma$ of the general divisor of the $g_{4}^{1}$ spans a line, whereas $e=1$ implies that the image on $\Gamma$ of the general divisor of the $g_{4}^{1}$ spans a line or a plane.

Our next result only works in $\mathbf{P}^{3}$. It provides our main tool for handling the function $\Delta_{D}^{2}$.

Theorem 2.3 (Descent Lemma). Let $\Gamma$ be a curve in $\mathbf{P}^{3}, \sigma: C \rightarrow \Gamma$ its normalization, $D$ a divisor on $C$.

If $t$ is any number which satisfies

$$
\left(\begin{array}{c}
t-1 \\
2
\end{array}\right)<\Delta_{D}^{1}(n)<d-\left(\begin{array}{l}
t \\
2
\end{array}\right)
$$

and $h^{0} \mathcal{I}_{\Gamma}(t-1)=0$, then we have $\Delta_{D}^{2}(n+1) \geq t$.

Proof. For a general point $\eta \in\left(\mathbf{P}^{3}\right)^{*}$ call $\pi_{\eta}$ the corresponding plane and let $Z_{\eta}$ be the pull-back on $C$ of $\pi_{\eta}$. For all $n$ we have an exact sequence:

$$
0 \rightarrow H^{0} \mathcal{O}_{C}(D+(n-1) H) \rightarrow H^{0} \mathcal{O}_{C}(D+n H) \rightarrow H^{0} \mathcal{O}_{Z_{\eta}}
$$

Call $\rho_{\eta, n}$ the last map and put $W_{\eta, n}=\operatorname{Im}\left(\rho_{\eta, n}\right)$, so that $\Delta_{D}^{1}(n)=\operatorname{dim} W_{\eta, n}$.

Choose now $m \gg 0$ and pick a general element $E \in|(m-n) H-D|$. We may identify $Z_{\eta}$ and $E$ with their images on $\Gamma$ via $\sigma$. Surfaces of degree $m$ through $E$ cut on the general plane $\pi_{\eta}$ a linear system whose restriction to $H^{0} \mathcal{O}_{Z_{\eta}}$ surjects onto $W_{\eta, n}$. It follows that around a general point $\eta$ of $\left(\mathbf{P}^{3}\right)^{*}$ we may find an étale neighbourhood $U$ and a subbundle $\mathcal{V}$ of the $m$-th power of the tautological bundle over the incidence variety in $\mathbf{P}^{3} \times U$, such that $\operatorname{rank}(\mathcal{V})=\Delta_{D}^{1}(n)$ and for any $\eta \in U$ the fiber $\mathcal{V}_{\eta, n} \subset H^{0} \mathcal{O}_{\pi_{\eta}}(n)$ restricts isomorphically to $W_{\eta, n}$ in $H^{0} \mathcal{O}_{Z_{\eta}}$.

By assumptions and by construction, $\mathcal{V}_{\eta}$ satisfies the hypothesis of [CCD2], proposition 4 , so that, for a general $\eta \in U$,

$$
\mathcal{V}_{\eta, n} \otimes H^{0} \mathcal{O}_{\pi_{\eta}}(1) \rightarrow H^{0} \mathcal{O}_{Z_{\eta}}
$$

has rank $\geq \operatorname{dim} W_{\eta, n}+t$.

It follows that the restriction map $H^{0} \mathcal{O}_{C}(D+(n+1) H) \rightarrow H^{0} \mathcal{O}_{Z_{\eta}}$ has rank $\geq \operatorname{dim} W_{\eta, n}+t=\Delta_{D}^{1}(n)+t$, which gives the claim.

For curves in higher dimensional spaces, we do not have such a precise result about the descent of the Hilbert function of $D$. Instead, we can use ideas from [CCD1] to show the following:

Proposition 2.4. Let $\Gamma$ be a curve in $\mathbf{P}^{r}, \sigma: C \rightarrow \Gamma$ its normalization, $D$ a divisor on $C$. Assume $\Delta_{D}^{2}(n)<r-1$ for some fixed $n$; then either $\Delta_{D}^{2}(i)$ vanishes for all $i<n$ or it vanishes for all $i>n$.

Proof. As before, consider the exact sequence

$$
0 \rightarrow H^{0} \mathcal{O}_{C}(D+(n-1) H) \rightarrow H^{0} \mathcal{O}_{C}(D+n H) \rightarrow H^{0} \mathcal{O}_{Z},
$$

where $Z$ is the pull-back to $C$ of a general hyperplane section of $\Gamma$; call $\rho_{n}$ the last map and put $W_{n}=\operatorname{Im}\left(\rho_{n}\right)$, so that $\Delta_{D}^{1}(n)=\operatorname{dim} W_{n}$. We may identify $Z$ with its image on $\Gamma$. 
Arguing as before, it is not hard to show that, for a general choice of the hyperplane, the points of $Z$ are in uniform position with respect to $W_{n}$; it follows by [CCD1], corollary 1.2 , that when $W_{n-1} \neq 0$ and $W_{n} \neq H^{0} \mathcal{O}_{Z}$, then $\operatorname{dim} W_{n} \geq \operatorname{dim} W_{n-1}+r-1$, i.e. $\Delta_{D}^{2}(n) \geq r-1$.

On the other hand, observe that $W_{n-1}=0$ implies $h^{0} \mathcal{O}_{C}(D+(n-2) H)=$ $h^{0} \mathcal{O}_{C}(D+(n-1) H)$, and this is possible only if $\phi_{D}(n-1)=0$; hence it gives $\phi_{D}(i)=0$ for all $i<n$. When $W_{n}=H^{0} \mathcal{O}_{Z}$ then $\Delta_{D}^{1}(n)=d$; hence $\Delta_{D}^{1}(i)=d$ for all $i \geq d$, and the claim follows.

\section{Numerical Results}

Definition 3.1. For all $m \in \mathbf{N}$ there are uniquely defined numbers $T(m)$ and $R(m)$ with $0 \leq R(m) \leq T(m)$ such that

$$
m=\frac{T(m)}{2}(T(m)+1)+R(m)=\left(\begin{array}{c}
T(m)+1 \\
2
\end{array}\right)+R(m) .
$$

Let us now turn our attention to non-negative numerical functions $F: \mathbf{Z} \rightarrow \mathbf{Z}$ which satisfy the following condition $(*)$ :

$$
F(n)=0 \text { for }|n| \gg 0, \text { and, putting } d=\sum F(i), \text { we have: }
$$

$$
F(n)<t \leq s \text { implies either } \sum_{i=-\infty}^{n-1} F(i) \leq\left(\begin{array}{c}
t-1 \\
2
\end{array}\right) \text { or } \sum_{i=-\infty}^{n-1} F(i) \geq d-\left(\begin{array}{l}
t \\
2
\end{array}\right) \text {. }
$$

The reason for studying these functions is clear from theorem 2.3: $\Delta_{D}^{2}$ is one of them. We are going to define a function $\Delta$ which minimizes the second sum among the functions satisfying $(*)$.

Definition 3.2. For given numbers $N, s, d$ with $d>N \geq 0$ and $d>s^{2}-s$, define $T, R, \rho, \epsilon$ as follows:

$$
\begin{gathered}
\quad T=\min (T(N), s-1), \\
\qquad \begin{array}{ll}
d=R(N), & \\
d-N-2=\frac{s}{2}(s+1)+\rho s+\epsilon & \text { if } \quad T(N) \leq s-1, \\
\rho=T(d-N-1)-s \quad \text { and } & d-N-1 \geq \frac{s}{2}(s+1), \\
\epsilon=R(d-N-1)+s-T(d-N-1)-3 & \text { otherwise },
\end{array}
\end{gathered}
$$

where $s>\epsilon \geq 0$.

Define the function $\Delta(n)=\Delta_{N, s, d}(n)$ as follows:

$$
\begin{cases}0, & n<0 \\ N+1, & n=0\end{cases}
$$


while for $n$ positive:

$$
\begin{cases}T+n+1, & 0<n \leq s-T-2, \\ s, & s-T-1<n \leq s-T+\rho+1, \\ 2 s-(n+T-\rho-1), & s-T+\rho+1<n \leq s-T+\rho+\epsilon+1, \\ 2 s-(n+T-\rho), & s-T+\rho+\epsilon+1<n \leq 2 s-T+\rho, \\ 0, & \text { elsewhere }\end{cases}
$$

(observe that some of the previous interval may be empty).

When $T(N) \leq s-1$, the function $\Delta$ has the following graph:

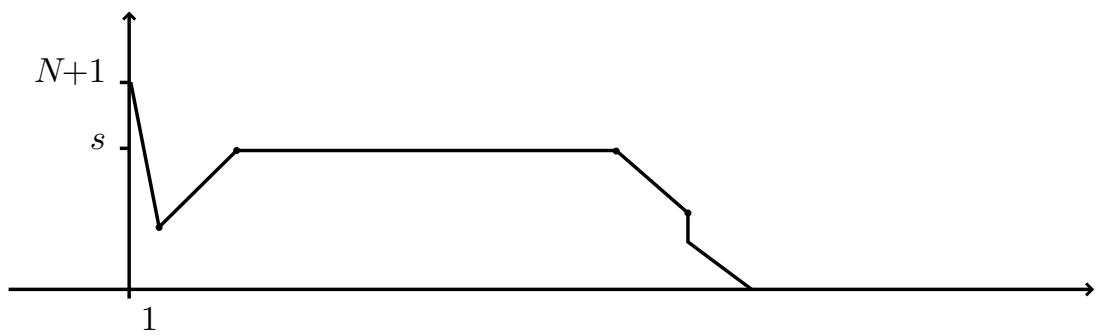

We put here a list of properties of the function $\Delta$ that we are going to use later. Their proofs are easy consequences of the definition.

Proposition 3.3. For all $n>0$ :

a) $0 \leq \Delta(n) \leq s$.

b) $\sum_{i=0}^{+\infty} \Delta(i)=d$.

c) $\sum_{i=n}^{+\infty} \Delta(i)>\left(\begin{array}{c}\Delta(n) \\ 2\end{array}\right)$, and hence $\sum_{i=0}^{n-1} \Delta(i)<d-\left(\begin{array}{c}\Delta(n) \\ 2\end{array}\right)$.

d) $\sum_{i=0}^{n-1} \Delta(i) \geq\left(\begin{array}{c}\Delta(n) \\ 2\end{array}\right)>\left(\begin{array}{c}\Delta(n)-1 \\ 2\end{array}\right)$.

e) For $n \leq s-T+\rho$ we have $\sum_{i=1}^{n} \Delta(i) \leq d-\left(\begin{array}{l}s \\ 2\end{array}\right)$.

f) If $N^{\prime} \geq N$ then, for all $n \geq 0$,

$$
\sum_{i=0}^{n} \Delta_{N^{\prime}, s, d}(i) \geq \sum_{i=0}^{n} \Delta_{N, s, d}(i) .
$$

Theorem 3.4. Let $F$ be a non-negative function which satisfies condition $(*)$. Put $\sum F(i)=d ;$ fix $N \geq 0$ and assume

$$
\sum_{i=-\infty}^{n_{0}} F(i) \geq \sum_{i=-\infty}^{n_{0}} \Delta_{N, s, d}(i)
$$

for some $n_{0} \geq 0$. Then for all $n \geq n_{0}$

$$
\sum_{i=-\infty}^{n} F(i) \geq \sum_{i=0}^{n} \Delta(i)
$$

and equality holds if and only if equality holds in $(+)$ and $F=\Delta$ from $n_{0}+1$ on.

Proof. If the claim does not hold, take $c \geq n_{0}$ minimal such that

$$
\sum_{i=-\infty}^{c} F(i)<\sum_{i=0}^{c} \Delta(i)
$$


Necessarily, by the minimality of $c$, we have

$$
\sum_{i=-\infty}^{c-1} F(i) \geq \sum_{i=0}^{c-1} \Delta(i) \quad \text { and } \quad F(c)<\Delta(c) \leq s
$$

hence by assumption we get either

$$
\sum_{i=0}^{c-1} \Delta(i) \leq \sum_{i=-\infty}^{c-1} F(i) \leq\left(\begin{array}{c}
F(c) \\
2
\end{array}\right) \leq\left(\begin{array}{c}
\Delta(c)-1 \\
2
\end{array}\right)
$$

which is excluded by proposition $3.3 \mathrm{~d}$ ), or

$$
\sum_{i=-\infty}^{c-1} F(i) \geq d-\left(\begin{array}{c}
F(c)+1 \\
2
\end{array}\right) \geq d-\left(\begin{array}{c}
\Delta(c) \\
2
\end{array}\right)
$$

Then it follows by $3.3 \mathrm{c})$ that

$\sum_{i=-\infty}^{c} F(i) \geq d-\left(\begin{array}{c}F(c)+1 \\ 2\end{array}\right)+F(c) \geq d-\left(\begin{array}{c}\Delta(c) \\ 2\end{array}\right)-1+\Delta(c) \geq \sum_{i=0}^{c-1} \Delta(i)+\Delta(c)$,

a contradiction.

The second claim is obvious.

Corollary 3.5. Under the previous assumptions, for $n \geq n_{0}$

$$
\sum_{j=-\infty}^{n} \sum_{i=-\infty}^{j} F(i) \geq \sum_{j=0}^{n} \sum_{i=0}^{j} \Delta(i)
$$

and equality implies $F=\Delta$.

Proof. The first assertion is a consequence of theorem 3.4. For the second, we note that if the equality holds, then certainly $F(n)=\Delta(n)$ for $n>n_{0}$, by theorem 3.4, and in the interval $(-\infty, 0), \Delta$ has the smallest second sum among the numerical functions with sum $N+1$.

\section{Applications to linear series}

In this section, $\Gamma$ is an irreducible, nondegenerate curve in $\mathbf{P}^{3}, \sigma: C \rightarrow \Gamma$ is its normalization, and $h^{0} \mathcal{I}_{\Gamma}(s-1)=0$ for a fixed number $s$ with $d>s^{2}-s$.

Computing the function $\Delta$ above, one is able to produce several results about linear series on $C$. Indeed, we have

Proposition 4.1. Let $\Gamma, C$ be as above, let $D$ be a divisor on $C$, and assume that $D$ is $H$-primary and $h^{0} \mathcal{O}_{C}(D)=N+1$. Then, for all $n>e(D)$,

$$
\delta \geq \sum_{j=-\infty}^{n} \sum_{i=-\infty}^{j} \Delta(i)+g-n d-1
$$

with equality if and only if $\Delta_{D}^{2}$ coincides with $\Delta_{N, s, d}$.

Proof. The function $\Delta_{D}^{2}$ satisfies condition $(*)$ of the previous section, so if we consider the corresponding function $\Delta:=\Delta_{N, s, d}$, by theorem 3.4 we know that its second sum bounds from below the Hilbert function of $D$ from 0 on. The assertion follows by Riemann-Roch. 
In general, the previous proposition leads to (lower) bounds for the degree of $H$-primary linear series of given dimension.

For instance, if one looks for the gonality of $C$, then one considers linear series $|D|$ with $\phi_{D}(0)=2$ and $\phi_{D}(n)=0$ for $n$ negative.

In order to apply proposition 4.1 , one needs to estimate the number $e(D)$. It is not hard to find numbers $n$ which satisfy $n>e(D)$ : it is enough to observe, for instance, that $e(D) \leq e \leq(2 g-2) / d$.

With more precise information on the level of speciality of $D$, one can expect better estimates on the degree of $D$.

In any event, a reasonable estimate for $e(D)$ is given by the following:

Proposition 4.2. Put $N=h^{0} \mathcal{O}_{C}(D)-1$ and define $T, \rho$ as in the previous section. Then

$$
h^{1} \mathcal{O}_{C}(D+n H)=0 \quad \text { for } n \geq 2 s-T+\rho-1 .
$$

Proof. For all $n \geq 2 s-T+\rho-1$, we have, by theorem 3.4 ,

$$
\Delta_{D}^{1}(n)=\sum_{i=-\infty}^{n} \Delta_{D}^{2}(i) \geq \sum_{i=0}^{n} \Delta_{N, s, d}(i)=d .
$$

The claim follows by proposition 1.4.

Remark 4.3. When $D=0$, we may write the previous function $\Delta_{N, s, d}$, starting from $\Delta(0)=1$. It is easy to see that in this case $\Delta$ is the function described in [H1], which leads to the computation of Halphen's bound $G(d ; s)$ for the genus of curves of degree $d>s^{2}-s$, not lying on any surface of degree $<s$. Recall that

$$
G(d ; s)=\frac{d^{2}}{2 s}+\frac{d(s-4)}{2}+1+\frac{\epsilon}{2}\left(s-\epsilon-1+\frac{\epsilon}{s}\right),
$$

where $d=k s-\epsilon$, with $0 \leq \epsilon \leq s-1$.

In other words, by applying propositions 4.1 and 4.2 above, for $D=0$, one finds the usual Halphen's bound for the genus of space curves not contained on surfaces of degree $<s$.

Using proposition 4.2 , and computing the second sum of $\Delta$, up to $2 s-T+\rho$, one gets

Theorem 4.4. Any $H$-primary linear series $g_{\delta}^{r}$ on $C$ satisfies

$$
\begin{aligned}
\delta \geq & g+(T-\rho-2 s) d-1+(\rho+1)\left[R-T+\frac{3 s^{2}+s}{2}+\frac{s}{2}(\rho+2)\right] \\
& -\frac{(s-\epsilon)(s-\epsilon-1)}{2}+s^{3}+\frac{3}{2} s^{2}+\frac{s}{2}-2 s T+2 s R-R T-\frac{T^{3}-3 T^{2}+2 T}{6},
\end{aligned}
$$

where $R, T$ are defined as above.

This bound is sharp, as we shall see soon.

Since any linear series of dimension 1 or 2 is $H$-primary, one gets

Corollary 4.5. Let $\Gamma$ be an irreducible space curve of degree $d$ and geometric genus $g$; assume that it does not lie on surfaces of degree $<s$, and let $d>s^{2}-s$. Call $C$ a normalization of $\Gamma$. 
If $D$ is a divisor of degree $\delta$ on $C$, defining a linear series $g_{\delta}^{1}$, then $N=1, T=1$, $R=0$ and

$$
\delta \geq g-s^{3}+\frac{3}{2} s^{2}+\frac{3}{2} s-\frac{3}{2} \rho s^{2}-s \epsilon+2 \rho s+\frac{\epsilon}{2}-\frac{\epsilon^{2}}{2}-\frac{s}{2} \rho^{2}-\rho \epsilon-\rho-2 .
$$

If $D$ is a divisor of degree $\delta$ on $C$, defining a linear series $g_{\delta}^{2}$, then $N=2, T=1$, $R=1$ and

$$
\delta \geq g-s^{3}+\frac{3}{2} s^{2}+\frac{3}{2} s-\frac{3}{2} \rho s^{2}-s \epsilon+2 \rho s+\frac{\epsilon}{2}-\frac{\epsilon^{2}}{2}-\frac{s}{2} \rho^{2}-\rho \epsilon-\rho-1
$$

(clearly $\epsilon$ and $\rho$ differ in the two expressions).

Let us discuss some examples.

Example 4.6. When $C=\Gamma$ is a smooth complete intersection of surfaces of degree $s$ and $p$ with $p \geq s(\geq 2)$, we find from the previous expression that, if $|D|$ is a $g_{\delta}^{1}$ on $C$, then

$$
\delta \geq s p-p .
$$

This is exactly the bound found in [B] (see also [CL] and [L], p. 23).

This bound is attained when $C$ has a $p$-secant line $\ell$ and $D$ is cut out by the planes through $\ell$, off the points cut out by $\ell$ on $C$. In fact, this is the unique kind of $g_{s p-p}^{1}$ on $C$. Indeed, since $D$ satisfies $\Delta_{D}^{2}(n)=\Delta(n)$ for all $n$, by 1.5 one computes $h^{1} \mathcal{O}_{C}(D+(s+p-5) H)>0$. So $\omega_{C} \otimes \mathcal{O}_{C}(-(s+p-5) H-D) \simeq \mathcal{O}_{C}(H-D)$ is effective, and clearly $H-D$ is contained in 2 independent planes.

Similarly, for a $g_{\delta}^{2}$ one has

$$
\delta \geq s p-1
$$

and the minimum is attained when the divisors lie on a plane, i.e. when the series is cut by the planes through a point.

Next, we apply 4.4 to Halphen curves.

Theorem 4.7. Let $C=\Gamma$ be a smooth Halphen curve of genus $g=G(d ; s)$, that is, a curve of degree $d>s^{2}-s$ and maximal genus among those not lying on surfaces of degree $<s$. Put $d-1=m s+e, 0 \leq e<s$, and assume that $C$ is not a complete intersection, i.e. $e \neq s-1$.

Then any linear series $g_{\delta}^{1}$ on $C$ satisfies $\delta \geq m s-m$, and this bound is sharp: it is attained if and only if $C$ has an $(m+e+1)$-secant line $\ell$, by the series $|H-C \cdot \ell|$, where $H$ is cut out on $C$ by a plane.

For any linear series $g_{\delta}^{2}$, we have $\delta \geq d-1$. Also this bound is sharp; if either $s>3$ and $m>2$ or $e<s-2$, then every $g_{d-1}^{2}$ on $C$ is cut out by the planes through a fixed point.

Proof. We know that $C$ is directly linked to a plane curve $C^{\prime}$ of degree $s-e-1$ by surfaces of degree $s$ and $m+1$ (see [H1]).

The two bounds are a straightforward consequence of theorem 4.4; for the sharpness, one has only to observe that there are Halphen curves with an $(m+e+1)$-secant line: they can be constructed as the residual of a plane curve of degree $s-e+1$ containing a line, in a complete intersection of type $(s, m+1)$.

It remains only to classify series of minimal degree.

Assume that a divisor $D$ defines a $g_{m s-m}^{1}$ on a smooth Halphen curve $C$; then we only need to prove that $H-D$ is effective, for then it must be contained on a line, because $C$ is arithmetically normal. 
The canonical series is cut on $C$ by surfaces of degree $s+m-3$ passing through $C^{\prime}$ and, by theorem 3.4, we know the function $\Delta_{D}^{2}$, which must coincide with the corresponding $\Delta_{1, s, d}$. Put $E=C \cdot C^{\prime}$ and consider the following cases:

(i) $e=s-2$; as in the previous example, computing $\Delta_{D}^{2}$ one finds that $h^{1} \mathcal{O}_{C}(D+(s+m-4) H)>0$, which, by Serre duality, means that $H-D-E$ is effective.

(ii) $e=s-3$; arguing as above, one gets here $h^{0} \mathcal{O}_{C}(2 H-D-E) \geq 2$, and since $C^{\prime}$ has degree 2 , it follows that there is a quadric containing $D$ and containing the plane of $C^{\prime}$; so $D$ must lie on a plane.

(iii) $e<s-3$; in this case $h^{1} \mathcal{O}_{C}(D+(s+m-5) H)>0$, so $2 H-D-E$ is effective; since $\operatorname{deg}\left(C^{\prime}\right)>2$, every quadric through $E$ contains the plane of $C^{\prime}$, and so $H-D$ is effective.

This complete the proof of the claim for the $g_{\delta}^{1}$ 's.

Take now a divisor $D$ which defines a $g_{d-1}^{2}$ on $C$. As above, since $\Delta_{D}^{2}=\Delta_{2, s, d}$, one finds that $h^{0} \mathcal{O}_{C}(2 H-D-E)>0$; hence there is an effective divisor in $|2 H-D-E|$, which we denote again by $2 H-D-E$. Consider the following cases:

(i) $e<s-3$; here $C^{\prime}$ has degee $>2$, and hence any quadric through $D+E$ contains the plane of $C^{\prime}$, so $H-D$ is effective and we are done.

(ii) $e=s-3 ; C^{\prime}$ is a conic and the effective divisor $2 H-D-E$ imposes 2 conditions on $|2 H-E| \supset(H-E)+|H|$. If $2 H-D-E$ has no common points with $H-E$, then it imposes 2 conditions on $|H|$; hence it must span a line, which is impossible for degree reasons. If $2 H-D-E$ has a common point with $H-E$, then any quadric through $D+E$ contains the plane of $C^{\prime}$; hence $H-D$ is effective.

(iii) $e=s-2$; then $C^{\prime}$ is a line and $h^{0} \mathcal{O}_{C}(2 H-E)=7$, and one computes $h^{0} \mathcal{O}_{C}(2 H-D-E)=2$. Assume that $|2 H-D-E|$ is base point free; then it cannot impose 4 conditions on $H$, as otherwise by Castelnuovo's lemma (see [C1]) it would impose at least 5 conditions on $2 H-E$, a contradiction, since this would imply $h^{0} \mathcal{O}_{C}(D) \leq 2$; on the other hand, if $2 H-D-E$ lies in some plane, then $|2 H-D-E|$ is cut by the planes through a fixed $(m+s-2)$-secant line; when $s>3$ and $m>2$ this line cannot exist, because $C^{\prime}$ is an $(m+s-1)$-secant and $C \cup C^{\prime}$ is a complete intersection of type $m+1, s$. Hence $|2 H-E-D|$ has a fixed point $P$, i.e. $|2 H-E-D|=P+|F|$, where $|F|$ is a $g_{m s-m}^{1}$; as we saw above, $|F|$ must be cut by the planes through an $(m+s-1)$-secant line, which must be $C^{\prime}$ when $s>2$; it follows that $H-D$ is effective.

Notice that the hypothesis $s>3$ and $m>2$, i.e. the hypothesis that the curve does not lie on a cubic surface, in the statement of theorem 4.7 above is necessary: for instance, a smooth curve of genus 2 and degree 5 is a Halphen curve, the complete intersection of a quadric and a cubic surface off a line, and it has a 2-dimensional family of $g_{4}^{2}$ on it, so the general such series is not cut out by planes through a point of the curve. In general, any smooth Halphen curve $C$ of degree $d=3 m+2$ on a cubic surface has linear series $g_{d-1}^{2}$ which are not cut out by planes: namely, as the proof of theorem 4.7 shows, any series of the type $\left|H-E+E^{\prime}\right|$, where $E$ is cut out on $C$ by the $(m+2)$-secant line and $E^{\prime}$ is cut out on $C$ by another line on the cubic.

Halphen curves are not the only curves which attain the bound of corollary 4.4. The curves in the next example are not even arithmetically normal.

Example 4.8. Let $C=\Gamma$ be a smooth curve which is directly linked to a pair $Y$ of disjoint lines, by surfaces of degree $s, p$, with $2<s \leq p$. Then we have $d=s p-2$, 
$g=-1+(s+p-4)(s p-4) / 2$ and $C$ is not contained on surfaces of degree $<s$. For a $g_{\delta}^{1}$ on $C$ one must have, by corollary $4.2, \delta \geq p s-p-s$.

On the other hand, it is not hard to compute that both lines of $Y$ are $(s+p-2)$ secant to $C$; hence the set of planes through any of them cuts on $C$ a $g_{p s-p-s}^{1}$.

Arguing as in the proof of theorem 4.7, we find that these series are the unique pencils of minimal degree on $C$. We omit the details.

For curves of relatively low genus with respect to the degree, the estimate of $e(D)$ given by proposition 4.2 is not sharp. This affects the sharpness of the bounds given in 4.4 and 4.5 too; so in order to improve them, one has to improve the estimate on $e(D)$.

Let us show, for example, what happens for curves on a smooth quadric.

Example 4.9. Let $Q$ be a smooth quadric surface and let $C$ be a curve of type $(a, b)$ on $Q$, with $a \leq b$. Then we know that $d=a+b, g=a b-a-b+1$, $e(0)=a-2$ and $s=2$. If $D$ defines a $g_{\delta}^{1}$ on $C$, the corresponding function $\Delta$ can be easily computed: it is a sequence of 2 's from 0 to $\left[\frac{d}{2}\right]$ with a final 1 if $d$ is odd, and 0 elsewhere.

Using corollary 4.5, one computes $\delta \geq a$, which is the expected result.

Since we know $\omega_{C}$, we can prove as above that for $a<b$, a series of minimal degree must be cut by a ruling of $Q$.

For a $g_{\delta}^{2}$, as above, one shows similarly that, for $a<b$, then $\delta \geq 2 a$, with the minimum attained only by the series cut by a pair of lines in a ruling; when $a=b$, the bound is $\delta \geq d-1$ and, as above, the minimum is attained by the series cut by the planes through a point.

Remark 4.10. The bounds that one finds for $\delta$ in the case $e(D) \geq s-T-1$ are strictly increasing with $s$. It follows that, for fixed genus $g$ and degree $d>s^{2}-s$ of $\Gamma$, the linear series attaining these bounds must live on curves lying on surfaces of degree $s$.

Example 4.11. Using our bound for computing the minimal degree of an $H$ primary $g_{\delta}^{3}$ on a Halphen curve $C$, one gets

$$
\delta \geq 2 d-6
$$

which is still sharp: look at the series cut by quadrics through 6 general points of $C$. In particular, when $d>6$, not every $g_{d}^{3}$ on $C$ can be $H$-primary.

This last fact leads to the following result, which should be compared with theorem 3.1 from $[\mathrm{CL}]$ :

Proposition 4.12. If $C$ is a smooth Halphen curve of degree $d>6$, i.e. a curve of maximal genus among those not lying on surfaces of degree $<s$, for some $s$ with $d>s^{2}-s$, then $|H|$ is the unique $g_{d}^{3}$ on $C$.

In other words, there is at most one way to embed a curve in $\mathbf{P}^{3}$ as a Halphen curve of degree $d>6$.

When the linear series $g_{\delta}^{N}$ is not $H$-primary, one gets lower bounds for $\delta$ using corollary 3.5 .

Proposition 4.13. Let $D$ define $a g_{\delta}^{N}$ on $C$ and let

$$
Q=\max \left\{n: N+1 \geq \sum_{j=0}^{n} \sum_{i=0}^{j} \Delta_{0, s, d}(i)\right\} .
$$


Then, for all $n>e(D)$,

$$
\delta \geq g-n d-1+\sum_{j=0}^{Q+n} \sum_{i=0}^{j} \Delta_{0, s, d}(i),
$$

with equality if and only if $N+1=\sum_{j=0}^{Q} \sum_{i=0}^{j} \Delta_{0, s, d}(i)$ and $\Delta_{D}^{2}(i)=\Delta_{0, s, d}(i-Q)$.

In particular,

$$
\delta \geq g-(2 s+\rho-Q-1) d-1+\sum_{j=0}^{2 s+\rho-1} \sum_{i=0}^{j} \Delta_{0, s, d}(i) .
$$

Proof. By our assumptions, there exists some number $c$, with $-Q \leq c \leq 0$, such that

$$
\Delta_{D}^{1}(c) \geq \sum_{i=0}^{c+Q} \Delta_{0, s, d}(i) .
$$

If we define $F(i)=\Delta_{D}^{2}(i-Q)$, then

$$
\sum_{i=-\infty}^{c+Q} F(i) \geq \sum_{i=0}^{c+Q} \Delta_{0, s, d}(i),
$$

and the first assertion follows by corollary 3.5 .

For the second, just note that, by construction,

$$
\sum_{i=0}^{2 s+\rho-1} \Delta_{0, s, d}(i)=d
$$

Computing this bound for the case of a $g_{\delta}^{3}$ on a Halphen curve, one gets $\delta \geq d$, so the bound is sharp, but we do not expect sharpness in more general situations.

Remark 4.14. It is possible to analyse other classes of space curves with the method we used here, but the computations often become quite cumbersome.

For example, one can consider smooth arithmetically Cohen-Macaulay curves with given arithmetical characters of the Hilbert-Burch resolution of their coordinate ring. In this case one can use the bound on $e$ given in [CGO], in order to get bounds for the degree of primary linear series. Once these computations have been performed, the problem of sharpness remains. However, at least for linear series of dimension $N \leq 3$, we expect the answer to this problem to be the natural one. For example, for $N=3$, we already know, from [CL], that in general there is no nonprimary $g_{d}^{3}$. Similarly, we expect that curves with minimal gonality should in general be obtained by a direct linkage to a curve containing a line.

\section{Results in $\mathbf{P}^{r}$}

When the curve sits in $\mathbf{P}^{r}$ we can obtain, using proposition 2.4, a bound for the degree of a linear series of given dimension. Since we are unable to prove a precise descent lemma like theorem 2.3 , the bound we obtain will be sharp essentially only for curves lying on minimal scrolls. 
The non-negative numerical functions $F$ that we consider now are those satisfying the following condition:

(**) If $F(n)<r-1$, then either $F(m)=0 \forall m<n$, or $F(m)=0 \forall m>n$.

We define here the function $\Delta=\Delta_{N, d}^{r}$, for $d \geq r+N$, by setting

$$
\begin{gathered}
d-N-2=\rho(r-1)+\epsilon, \quad 0 \leq \epsilon<r-1, \\
\Delta(n)= \begin{cases}N+1, & n=0 \\
r-1, & 1 \leq n \leq \rho, \\
\epsilon+1, & n=\rho+1, \\
0, & \text { elsewhere. }\end{cases}
\end{gathered}
$$

We are able to reproduce easily all the previous arguments, obtaining

Theorem 5.1. Let $F$ be a non-negative function which satisfies condition (**). Put $\sum F(i)=d$; fix $N>0$ and assume

$$
\sum_{i=-\infty}^{n_{0}} F(i) \geq \sum_{i=0}^{n_{0}} \Delta_{N, d}^{r}(i)
$$

for some $n_{0} \geq 0$. Put $\Delta=\Delta_{N, d}^{r}$. Then, for all $n \geq n_{0}$,

$$
\sum_{i=-\infty}^{n} F(i) \geq \sum_{i=0}^{n} \Delta(i)
$$

and equality holds if and only if equality holds in $(++)$ and $F=\Delta$ from $n_{0}$ on. Also,

$$
\sum_{j=-\infty}^{n} \sum_{i=-\infty}^{j} F(i) \geq \sum_{j=0}^{n} \sum_{i=0}^{j} \Delta(i),
$$

and equality implies $F=\Delta$.

Moreover, assume that, for some $n_{0} \geq 0$,

$$
\sum_{j=-\infty}^{n_{0}} \sum_{i=-\infty}^{j} F(i)=\sum_{j=0}^{n_{0}} \sum_{i=0}^{j} \Delta_{0, s, d}(i) .
$$

Then, for all $n \geq n_{0}$

$$
\sum_{j=-\infty}^{n} \sum_{i=-\infty}^{j} F(i) \geq \sum_{j=0}^{n} \sum_{i=0}^{j} \Delta_{0, s, d}(i),
$$

with equality if and only if $F=\Delta$.

Finally, we have $e(D)<\rho$.

Turning to our curves $C$, we know by proposition 2.4 that $\Delta_{C}^{2}$ satisfies condition (**). By Riemann-Roch we have

Corollary 5.2. Assume that $D$ defines an $H$-primary $g_{\delta}^{N}$ on the normalization $C$ of a non-degenerate curve $\Gamma \subset \mathbf{P}^{r}$. Then, for all $n>e(D)$

$$
\delta \geq \sum_{j=-\infty}^{n} \sum_{i=-\infty}^{j} \Delta(i)+g-n d-1,
$$


with equality if and only if $\Delta_{D}^{2}=\Delta$. In particular,

$$
\delta \geq g-\epsilon \rho+N-\rho-\left(\rho^{2}-\rho\right) \frac{r-1}{2}
$$

Example 5.3. If we apply corollary 5.2 to find the minimal degree for $H$-primary linear series $g_{\delta}^{N}$ on Castelnuovo curves in $\mathbf{P}^{r}$, then we find in many cases the expected value (see $[\mathrm{A}]$ for the case of pencils).

Indeed, we recall (see [H2]) that such a curve is smooth and lies on a surface $S$ of minimal degree $r-1$; when $r>5$ and $S$ is smooth, then it is a scroll whose Picard group is generated by two classes $h, f$, where $f$ represents the ruling and $h$ the hyperplane class. In this case, Castelnuovo curves on $S$ are in the classes $a h-(r-2+b) f$, with $b=0, \ldots, r-2$, and for a $H$-primary $g_{\delta}^{N}$ the minimal value of $\delta$ is computed from the previous formula; one gets

$$
\delta \geq \begin{cases}a N, & b \geq N, \\ (a-1) N+b, & b<N .\end{cases}
$$

When $N=1$, this is the value found in $[\mathrm{A}]$; it is attained by the linear series cut by $f$. Furthermore, since we know the canonical class of $S, \omega_{S}=-2 h+(r-3) f$, we may also compute that a linear pencil of minimal degree, whose function $\Delta_{D}^{2}$ is then equal to $\Delta$, must be cut by the ruling. This is done as for the examples considered in $\S 4$.

For linear series of dimension $N=2, \ldots, r-1$ (necessarily they are $H$-primary) we find that the minimal degree is achieved when the series is a multiple of the ruling, in the case $b \geq N$, while for $b<N$ the bound is always attained, for $b=0$, on curves lying on a cone, by the $N$-th multiple of the linear series cut out by the ruling, off the vertex of the cone.

Furthermore, one can prove directly that the previous bound is always attained, for Castelnuovo curves and for all $N=2, \ldots, r-1$, when $r \leq 5$ (recall that in $\mathbf{P}^{5}$ one may also consider curves lying on a Veronese surface). On the other hand a smooth Castelnuovo curve $C$ in $\mathbf{P}^{6}$ of degree 12 has genus 7 , and the previous formula tells us that a $g_{\delta}^{2}$ on $C$ has degree $\delta \geq 5$. This is certainly true, but the bound cannot be sharp for there are no $g_{5}^{2}$ on a smooth curve of genus 7 , unless the curve is hyperelliptic, which is not our case.

The reason for this lies in the fact that our method uses a reduction to some hyperplane section of $C$; hence it is not sensitive to the isolated singularities of the curve; in the previous example, if we allow a node for $C$, then the genus becomes 6 and the formula tells us that $\delta \geq 4$ for a $g_{\delta}^{2}$; this bound is attained on the hyperelliptic curve of degree 12 which is residual of 3 rulings on a quintic cone. Notice that this curve has maximal arithmetic genus among nondegenerate curves in $\mathbf{P}^{6}$.

Example 5.4. When the curve $C$ lies on a minimal scroll but is not a curve of maximal genus, then the previous formulae are not sharp. One has to put into the picture the number $e(D)$, which is easily computed: we get $e(D)=a-2$ for a curve of type $a h+b f, b \geq 0$.

Using the function $\Delta$, one gets that the minimal gonality, for such a curve, is exactly $a$.

Example 5.5. When $N \geq r$, we have to distinguish the $H$-primary and the non$H$-primary cases and use theorem 5.1 (iii) for this last one. For instance, when $C$ is 
a Castelnuovo curve, as in example 5.3, for $N=r$ one finds that the minimal degree for a $g_{\delta}^{r}$ on $C$ is $\delta=d$, and the unique series of smallest degree is the hyperplane series. Indeed, for $H$-primary series we get $\delta \geq r a-r+b$, which is bigger than $d$.

\section{REFERENCES}

[A] Accola R., On Castelnuovo's inequalities for algebraic curves I, Transactions of Amer. Math. Soc. 251 (1979), 357-373. MR 80i:14008

[B] Basili B., Indice de specialité des groupes des points de l'espace, Bull. Soc. Math. France 124 (1996), 61-95. MR 97f:14048

[CCD1] Chiantini L., Ciliberto C., Di Gennaro V., The genus of projective curves, Duke Math. J. 70 (1993), 229-245. MR 94b:14027

[CCD2] Chiantini L., Ciliberto C., Di Gennaro V., The genus of curves in $\mathbf{P}^{4}$ satisfying certain flag conditions, Manuscr. Math. 88 (1995), 119-134. MR 96h:14044

[CCD3] Chiantini L., Ciliberto C., Di Gennaro V., On the genus of projective curves satisfying certain flag conditions, Boll. UMI (7) 10-B (1996), 701-732. MR 98g:14028

[C1] Ciliberto C., Alcune applicazioni di un classico procedimento di Castelnuovo, Sem. di Geometria 1982-83 - Univ. Bologna pages 17-43. MR 86j:14027

[CGO] Ciliberto C., Geramita A., Orecchia F., Remarks on a theorem of Hilbert-Burch, Boll.UMI (7)2-B (1988), 463-483. MR 90a:13037

[CL] Ciliberto C., Lazarsfeld R., On the uniqueness of certain linear series on the curves of some class, Springer Lecture Notes 1092 (1984), 198-213. MR 86b:14015

[CK] Coppens M., Kato, Non-trivial linear systems on singular plane curves., Mathematishce Nachr. 166 (1994), 71-82. MR 95k:14006

[Ha] Halphen G., Mémoire sur la classification des courbes gauches algébriques, J. Ec. Polytech. 52 (1882), 1-200. MR

[H1] Harris J., The genus of space curves, Math. Ann. 249 (1980), 191-204. MR 81i:14022

[H2] Harris J. (with the collaboration of D. Eisenbud), Curves in projective space, Montreal University Press (1982). MR 84g:14024

[L] Lazarsfeld R. (with the assistance of G.F. del Busto), Lectures on linear series, IAS/Park City Math. Series 3 (1993), Amer. Math. Soc., Providence, RI, 1997, pp. 161-219. MR 98h:14008

[M] Mezzetti E., Differential-geometric methods for the lifting problem and linear systems on plane curves, Journal of Algebraic Geometry 3 (1994), 375-398. MR 95d:14049

[P] Paoletti R., Seshadri positive curves in a smooth threefold, projective 3-fold, Atti Accad. Naz. Lincei Cl. Sci. Fis. Mat. Natur. Pend. Lincei (9) Mat. Appl. 6 (1995), 259-274. MR 97d:14049

Università di Siena, Dipartimento di Matematica, Via del Capitano 15, 53100 Siena, ITALY

E-mail address: chiantini@unisi.it

Università di Roma "Tor Vergata", Dipartimento di Matematica, Via della Ricerca Scientifica, 00133 Roma, Italy

E-mail address: cilibert@axp.mat.uniroma2.it 